\title{
COLLISIONAL QUENCHING AT ULTRALOW ENERGIES: CONTROLLING EFFICIENCY WITH INTERNAL STATE SELECTION
}

\author{
S. Bovino, E. Bodo and F.A. Gianturco* \\ Department of Chemistry and CNISM, University of Rome La Sapienza, \\ Piazzale A. Moro 5, 00185 Rome, Italy
}

\begin{abstract}
Calculations have been carried out for the vibrational quenching of excited $\mathrm{H}_{2}$ molecules which collide with $\mathrm{Li}^{+}$ions at ultralow energies. The dynamics has been treated exactly using the well known quantum coupled-channel expansions over different initial vibrational levels. The overall interaction potential has been obtained from the calculations carried out earlier in our group using highly correlated ab initio methods. The results indicate that specific features of the scattering observables, e.g. the appearance of Ramsauer-Townsend minima in elastic channel cross sections and the marked increase of the cooling rates from specific initial states, can be linked to potential properties at vanishing energies ( sign and size of scattering lengths) and to the presence of either virtual states or bound states. The suggestion is made that by selecting the initial state preparation of the molecular partners, the ionic interactions would be amenable to controlling quenching efficiency at ultralow energies.
\end{abstract}

\footnotetext{
* Corresponding author; e.mail address: fa.gianturco@caspur.it; fax: +39.06.49913305.
} 


\section{INTRODUCTION}

Manipulation of atomic and molecular systems at ultralow energies has completely changed the research landscape of experimental and theoretical molecular phenomena. Thus, intense efforts have been directed to the study of new properties of Bose-Einstein condensates [1, 2] and to analyze the quantum degeneracy features of Fermi gases like ${ }^{40} \mathrm{~K}$ [3] and ${ }^{6} \mathrm{Li}$ [4], since ultracold quantum matter containing fermions has exhibited a new range of unexpected properties. The occurrence of Bose-Einstein condensation and of Fermi degeneracy in diluted gases typically requires temperatures between $1 \mathrm{nK}$ and $1 \mu \mathrm{K}$, although new quantum effects can begin to show up already around a few mK, where the Broglie wavelengths often become large compared to the available atomic and molecular dimensions. Under such special environmental conditions the relevant collisions between partners, be them atomic or/and molecular, become fully quantal and are chiefly guided by the long-range interactions: the regions just below $1 \mathrm{mK}$ are usually opening up the ultracold collisional regimes.

Over the last few years, with the increase of the interest on molecular systems in the ultracold regime, and on the processes taking place in cold traps or in Coulomb crystals involving both neutral and ionic molecules, there has been a shift of focussing onto the details and features of possible means one can employ to control ultracold molecular quantum systems. The latter, in fact, show a much richer structure of internal energy levels with respect to atoms and offer more possibilities for quantum control. For example dipolar gases are predicted to exhibit new features which have likely applications in quantum computing [5, 6] and the possibility of manipulating their reactive and cooling efficiency from selected initial states still remains an intriguing process which is clearly linked to their structural features.

Furthermore, the direct process of cooling molecules from initial room temperature conditions by using precooled atomic gases is one of the mayor ways in which such special, ultracold systems could be prepared [7]. It therefore becomes important to be able to know just how efficient the cooling processes can be and how much they are influenced by the initial preparation of the molecular targets. The computational modelling of such assessments is, however, a rather complicated affair where one needs to know reliably the overall range of interaction between molecular partners, or between molecules and cold atoms, the detailed dynamical coupling matrix elements between that interaction and the rotovibrational man- 
ifold of at least one molecular partners and, finally, one should be able to solve exactly the coupled-channel (CC) quantum dynamics at the cold and ultracold temperature regimes. The last requirement, however, become easier to achieve under those special conditions since the quantum suppression effects strongly reduce the number of contributing angular momenta during the scattering event [10, 11]. Furthermore, the marked disappearance of quantum interference between trajectories (as only the s-wave contribution dominates the scattering S-matrix) enhances the nanoscopic features of each collision and suggests that ultimate control of the scattering attributes could be more easily detected when dealing with cold and ultracold scattering experiments [12] than in the case of room temperature dynamics.

The aim of the present work is therefore that of showing, in a specific molecular system where reactive channels can also become open at higher energies, the effect on scattering observables and on inelastic quenching cross sections of starting the collisional process with the molecular partner (the $\mathrm{H}_{2}$ molecule) prepared in a specific vibrational level. We had already studied the neutral $\mathrm{Li}_{2}+\mathrm{He}$ molecular system in the case of non reactive events at ultralow energies [13] and had found a marked dependence of cross section magnitudes on the initial vibrational channel, although the reaction examined involved a rather weak interaction among neutral partners. In the present study we shall analyse instead a collision process in an ionic system

$$
\mathrm{Li}^{+}+\mathrm{H}_{2}(v) \leftrightarrows \mathrm{Li}^{+}+\mathrm{H}_{2}\left(v^{\prime}<v\right)
$$

to see the dynamical behaviour when a stronger potential energy surface (PES) is involved, and a PES for which a reactive channels also opens up for $v>11$

$$
\mathrm{Li}^{+}+\mathrm{H}_{2}(v>11) \leftrightarrows \mathrm{LiH}^{+}+\mathrm{H}
$$

although we shall not be considering in the present work the additional contributions from such channels. We have already analyzed in a preliminary report [14] the quenching cross sections of reaction (1) for the lowest three vibrational states of $\mathrm{H}_{2}$. The aim of the present work is to extend the study to the full range of accessible initial states, thereby providing a more complete picture on the controlling capabilities of initial state preparation in molecular encounters.

The following Section 2 will therefore outline our scattering equations and their behaviour at ultralow energies, together with sketching the general features of the involved potential 
energy surface. The next Section 3 will present our results while Section 4 will summarize our conclusions.

\section{INTERACTION FORCES AND COLD DYNAMICS}

The present calculations employ the adiabatic electronic ground state of the $\mathrm{LiH}_{2}^{+}$complex at collision energies well below its barrier to the reaction of eq. (2) so that we can assume that the inelastic channels of the process (1) are the only ones which are open at the considered energies: the reaction of eq. (2) is, in fact, endothermic by about $4.4 \mathrm{eV}$ due to the lower binding energy of $\mathrm{LiH}^{+}$with respect to $\mathrm{H}_{2}$. It therefore shows a purely uphill route in going to the $\mathrm{LiH}^{+}$formation, with an absolute minimum energy geometry of the $\mathrm{Li}^{+} \mathrm{H}_{2}$ complex in $\mathrm{C}_{2 v}$ orientation. The details of the full reactive PES, together with those on the quality of the employed ab initio method to yield the raw points of the surface, have been discussed before [15, 16] and will not be repeated here. Suffice it to say that on the reagents' region of the $\mathrm{Li}^{+}$ion approaching the $\mathrm{H}_{2}$ molecule, the corresponding minimum energy path exhibits a well of about $2300 \mathrm{~cm}^{-1}$, which reduces to $\sim 650 \mathrm{~cm}^{-1}$ for the collinear orientations. That part of the interaction is dominated by the charge interacting with the molecular permanent quadrupole and by the spherical and non-spherical polarizabilities of the $\mathrm{H}_{2}(v)$ molecule. To briefly remind readers of its general features, the collinear profile of the reactive path is sketched in figure 1, where we also report the relative locations of the first eleven vibrational states of $\mathrm{H}_{2}$ existing below the barrier to $\mathrm{LiH}^{+}$formation, a process which we therefore will not be considering to occur at the ultralow collision energies examined in the present study.

One clearly sees in the figure that all collisions starting with vibrationally excited hydrogen molecules up to the 12th vibrational level, and occurring at vanishing energies, will be below barrier and will thus only access the inelastic channels of eq. (1). One should also keep in mind that the processes, which we shall be analyzing, occurring very closely to the near-zero energy region of the upper rim of the interaction potentials, will depend rather crucially on the efficiency of the potential couplings between target levels and therefore on the shape of the matrix elements given by convolutions over target asymptotic vibrational states $\varphi_{i}(r)$

$$
V_{i j}(R, \vartheta)=\left\langle\varphi_{j}|V(R, r, \vartheta)| \varphi_{i}\right\rangle
$$

which will therefore appear in the corresponding familiar $\mathrm{CC}$ formulation of the collision 
problem discussed below.

The vibrational wave functions and the vibrational levels of $\mathrm{H}_{2} \varphi_{i}=\varphi_{v j}$ are obtained by solving the Schrödinger equation for the diatom using the potential of ref. [20]. The corresponding coupled-channel equations in the space-fixed (SF) reference frame are then given by

$$
\left\{\frac{d^{2}}{d R^{2}}+\mathbf{k}^{2}-\mathbf{V}-\frac{\ell^{2}}{R^{2}}\right\} \mathbf{F}^{J}=0
$$

where, as usual, $\left[\mathbf{k}^{2}\right]_{i j}$ is the diagonal matrix for the asymptotic (squared) wave vectors, the coupling matrix $\mathbf{V}=2 \mu \mathbf{U}$ contains the full potential terms and $\ell^{2}$ is the matrix representation of the square of the relative orbital angular momentum: $\left[\ell^{2}\right]_{i j}=\delta_{i j} \ell_{i}\left(\ell_{i}+1\right)$. The matrix $\mathbf{F}^{J}$ holds the radial solutions for each choice of the total angular momentum $\mathbf{J}$, the dynamical constant of the motion for the present system.

In the asymptotic region the solution matrix can be written as

$$
\Psi(R)=\mathbf{J}(R)-\mathbf{N}(R) \cdot \mathbf{K}
$$

where $\mathbf{J}(R)$ and $\mathbf{N}(R)$ are diagonal matrices containing Riccati-Bessel and Riccati-Newmann functions and from which we obtain $\mathbf{K}$ and then $\mathbf{S}$. The corresponding state-to-state superelastic cross sections will be given by

$$
\sigma_{v j, v^{\prime} j^{\prime}}\left(E_{i}\right)=\frac{\pi}{(2 j+1) k_{v j}^{2}} \sum_{J}(2 J+1) \sum_{l, l^{\prime}}\left|\delta_{l v j, l^{\prime} v^{\prime} j^{\prime}}-S_{v j, v^{\prime} j^{\prime}}^{l l^{\prime}}\right|^{2}
$$

The corresponding expansion of the elastic matrix element for $\ell=0$ in powers of $k$ allows one to write [8, 9]

$$
S_{v j, v^{\prime} j^{\prime}} \simeq 1+2 i \delta_{v j}(k)=1-2 i k\left(\alpha_{v j}-\beta_{v j}\right)=1-2 i k a_{v j}
$$

which makes us obtain the real $\alpha_{v j}$ and imaginary $\beta_{v j}$ parts of the scattering length $a_{v j}$. The corresponding elastic and inelastic parts of the total scattering cross sections, in the same limit of $k \rightarrow 0$, are given by

$$
\sigma_{v j}^{e l}=4 \pi\left|a_{v j}\right|^{2}, \quad \sigma_{v j}^{i n .}=\frac{4 \pi \beta_{v j}}{k}
$$

We can further derive the behavior of quenching rates for a selected initial state, by relating it to the imaginary part of the scattering length or, equivalently, to the inelastic quenching cross section [8, 9]

$$
R_{v j}=\frac{4 \pi \hbar}{\mu} \beta_{v j}
$$


At ultralow collision energies the features of the scattering attributes as obtained from the computed S-matrix (i.e. the size and sign of the complex scattering length for each initial state) can tell us of the existence for the corresponding scattering potential energy surface of either zero-energy resonant states (virtual states) or of bound states supported very near the threshold energies depending on the sign of the real part of the complex scattering length, $\alpha_{v j}$. The (complex) energy of these states is given by:

$$
E=-\frac{\hbar^{2}}{2 \mu\left|a_{v j}\right|^{2}}\left(\cos 2 \gamma_{v j}+i \sin 2 \gamma_{v j}\right)=E_{v j}-\frac{i}{2} \Gamma_{v j}
$$

where $\gamma_{v j}=\tan ^{-1} \frac{\beta_{v j}}{\alpha_{v j}}$. Whenever a positive scattering length exists, one can see it to increase to infinity as $E$ approaches zero energy from below and to reappear at increasingly larger negative values as $E$ moves on the second Riemann's sheet as a virtual state of the ionic complex. The above quantities obviously depend on the interaction potential and hence on the features of the coupling matrix elements between vibrational levels involved in the quenching process. This is particularly so with ionic interactions where the longrange coupling in all channels could produce, even at ultralow energies, contributions to the opacity functions beyond the more usual s-wave contribution [9, 14, 17]. It is one of the aims of the present work to show that this is indeed the case in the present system and that the presence near the potential top of either bound states or virtual states strongly affects the quenching efficiency of the ultracold $\mathrm{H}_{2}$ molecules.

To give us a pictorial idea of the features of the coupling potential terms between vibrational levels of the target molecule, we report in figure 2 their radial shapes at two different orientations and between some of the vibrational levels: only diagonal terms are shown in the two panels of that figure.

One notices from the figure that the overall potential strength increases as the molecule is vibrationally more excited: the repulsive walls at $0^{\circ}$ move outwards as $v$ increases due to the stretching of the molecular bond. The physical consequances of such feature on the behavior of the quenching cross section will be further discussed in the next section.

\section{ELASTIC AND QUENCHING COLLISIONS}

As mentioned before, the aim of the present study is to extend our earlier dynamical study [14] on the feasibility of controlling the quenching efficiency of vibrationally excited 
$\mathrm{H}_{2}$ molecules, when colliding at ultralow energies with a simple molecular ion, to the full range of accessible vibrational levels in order to see which cross sections and rates exhibit some special behavior, that leads to larger relaxation rates or to special features in the elastic cross sections.

In order to describe the molecular target we have employed all the open vibrational channels for a specific initial state and three closed vibrational channels above them. Furthermore, for each vibrational level, up to $j_{\max }=12$ rotational channels were included in the expansion for a maximum of about 70 asymptotic coupled channels. The corresponding coupled equations were propagated from the inner region out to $500 \AA$ and the values of total angular momenta needed for convergence ranged from $J=0$ (at the lowest energies) up to $J=9$ (at the highest ones).

The computed elastic cross sections as a function of collision energy, on a log-log scale, are reported by figure 3 for each of the initial vibrational states in which the $\mathrm{H}_{2}$ molecule is being prepared (the rotational initial state is $j=0$ ).

From the data reported in that figure the following considerations could be made:

- at collision energies below $10^{-3} \mathrm{~cm}^{-1}$ one notices the linear dependence of the elastic cross sections on the energy and their being constant in value below $10^{-4} \mathrm{~cm}^{-1}$ : this means that we have reached the low-energy Wigner's law regime [18], whereby the $\ell=0$ contributions to the cross sections dominate the scattering and become energyindependent in the elastic channels.

- at the collision energies between $10^{-2}$ and $2 \cdot 10^{-3} \mathrm{~cm}^{-1}$ specific cross sections for the molecular target initially prepared in the $v=1,3$ and 9 vibrational states show a very marked drop. Such features are indicative of the presence of Ramsauer-Townsend (RT) minima, whereby the scattering process is mainly controlled by $s$-wave and the corresponding eigenphase for the $\ell=0$ channel vanishes [19]. The $s$-wave scattering length is negative and a virtual state can be present in the triatomic complex.

The fact that three different target preparations can lead to such marked features of the elastic channels at energies around the mK regime suggests that they could possibly be amenable to experimental detection, thereby providing a rather sensitive test on the quality of the ion-molecule interaction potential employed by our calculations. 
The differences which exist once the inelastic quenching cross sections are examined, could be gleaned by looking at the corresponding dominant partial wave contributions to inelastic channels. The results reported in the four panels of figure 4 show, in fact, the relative importance of two partial waves $(\mathrm{J}=0$ and $\mathrm{J}=1)$ for possibly selecting quenching cross sections for which the $\mathrm{H}_{2}$ molecule is prepared in a specific initial vibrational state $(v, \mathrm{j}=0)$ and decays into the $(v-1, \mathrm{j}=2)$ states.

One clearly sees that, for all the de-excitation processes presented in the panels, the $\mathrm{J}=0$ contributions are by far the dominant ones as one reaches the Wigner regime of the cross sections. On the other hand, in the collision energy range of the $10^{-3}$ wavenumbers one clearly sees the very strong additional contribution from the $\mathrm{J}=1$ ( $p$-wave) channels that suggest the presence of additional open channel resonances (dynamical trapping) induced by the strong ion-molecule long-range contributions to the scattering process.

We have also found similar $p$-wave contributions appearing for ultra-cold collisions for $\mathrm{H}_{2}$ molecules prepared in the $v=1$ and $v=2$ initial states only, and discussed it in our previous preliminary report [14]: we show here that such resonant features indeed extend up the vibrational ladder to the higher initial states presented by figure 4 . With the same token, the features of the elastic channels presented by figure 3 extend to the $v=9$ initial state of the molecular partner the RT minimum appearance already discussed for the $v=1$ and $v=3$ states [14].

The presence of marked $p$-wave contributions to the quenching cross sections around the $10^{-3} \mathrm{~cm}^{-1}$ energy regime will obviously affect the efficiency of the quenching process and therefore will be reflected in the corresponding behavior of the inelastic cross sections.

The calculations reported by figure 5 , in fact, show the behavior of the total inelastic cross sections (summed over all final ro-vibrational states) originating from a set of selected initial vibrational states of the molecular partner (the rotational initial state is $j=0$ ): the energy region goes from 0.1 wavenumber down to $10^{-6} \mathrm{~cm}^{-1}$.

The following comments could be made from a perusal of the reported data:

- the initial molecular preparation in the $v=7$, which had shown a strong $p$-wave resonant contribution in figure 4 , shows now the largest cooling efficiency in the milli-cm ${ }^{-1}$ energy region;

- the initial levels 6,8 and 4 show decreasing effects from $p$-wave contributions (see 
figure 4). Hence, their quenching cross sections within the same range of energies also show decreasing efficiency in going from $v=6$ to $v=8$ and to $v=4$;

The overall behavior of the scattering observables as a function of the prepared initial vibrational state is given by the numbers reported in Table 1. The quenching rates are given both as asymptotic values at vanishing temperature and as convoluted values around the millikelvin region (last column in Table). We clearly see that those initial states where resonant features were detected from $p$-wave contributions (see figure 4) are also showing the largest quenching rates across the same range of energies. This confirms that ionic interactions can be capable, even at ultralow temperatures, to cause dynamical trapping because of the $\ell=1$ barriers which appear to give substantial contributions to the cross section at those energies. However, although the importance of such effects is a strong function of the selected molecular target, the present results indeed suggest that internal energy preparation at ultracold collisions could be a possible path to controlling quenching efficiency of ionic systems.

A summary of the features which have been shown by the present calculations is pictorially given by the results we collect in the four panels of figure 6 .

From the data in the figure one sees that the rates in the Wigner's regime region show a constant increase as $v$ increases, thus suggesting, as already found in our earlier work on neutral systems [13], that when molecular targets have a low density of vibrational states per unit of energy the corresponding quenching efficiency has a strong dependence on the state preparation of the molecule. We further see that the $v=6$ state shows a very strong rate increase which is larger than that expected for it from the general trend of the values in the panel. This initial state exhibits both the presence of a shape resonance (see fig.4) and a fairly large and positive scattering length, a fact which suggests a bound state of the complex very near threshold, as confirmed by the data in the lower right panel of the same figure. Thus, one may argue that ionic interactions which are able to support metastable bound states for the triatomic complex could also provide increased quenching efficiency: this feature, in fact, is confirmed in figure 6 by the results provided by the $\mathrm{H}_{2}$ state preparations in the $v=6,7$ and 8 levels. We also note that negative scattering length values, i.e. signatures of virtual state formation, are present for $v=1,3$ and $9 \mathrm{H}_{2}$ states, exactly as indicated by the RT mimimum features of figure 3. 


\section{SUMMARY AND CONCLUSIONS}

In the present analysis we have extended our previous work [14] on the vibrational collisional quenching processes at low and ultralow energies for the subreactive channels of an ionic system, the $\mathrm{Li}^{+}+\mathrm{H}_{2}(v)$ case, to see if that collisional efficiency can be related both to features of a realistic interaction potential from ab initio calculations and to the specific internal energy content with which the target molecule is being prepared before undergoing a collisional quenching.

One important element at the millikelvin regime has been found to be the presence of non $s$-wave contributions to the opacity functions of the inelastic state-to-state vibrational channels. In other words, substantial $l=1$ contributions appear when specific vibrational initial states are considered, thereby causing shape resonances to occur and a marked increase of the quenching efficiency to appear. This is an important result which suggests that the reaction studied could be controlled via selecting specific initial states in the neutral molecule which in turn drive either up or down in size the corresponding vibrational quenching rates.

Furthermore, our study has shown that the presence of virtual states near threshold supported by the interaction potential is reflected at the millikelvin regime by the appearance of very marked elastic cross section minima which should be amenable to experimental observation and should then allow us to test the quality of the chosen potential energy surfaces, possibly modifying them according to experiments.

Finally, the quenching rates at vanishing collision energies, i.e. well into the Wigner's regime of scattering cross sections, indicate a strong dependence of their size on the selected initial state of $\mathrm{H}_{2}$ : they turn out to vary by more than two orders of magnitude when moving from the $v=1$ initial state to the $v=9$ state. The main cause of changes is seen to be related to the increased target size as $v$ increases, together with the additional appearance of either bound or virtual states of the compound system at energy very close to threshold. Both are important factors for driving upwards the quenching efficiency, as illustrated by the numbers of Table 1 and by the panels of figure 6 .

In conclusion, the present calculations for an ionic case in which both rotational and vibrational degrees of freedom of the molecular partner are described as coupled to the incoming ion via a fairly realistic PES [14, 16] indeed show a marked dependence of scattering observables on the initial rovibrational state in which the target molecule is being prepared 
and therefore suggest that the latter state-preparation could be providing a viable procedure for controlling the efficiency of the quenching process.

\section{Acknowledgments}

The financial support of the University of Rome Research Committee, of the CASPUR Computing Consortium, of the MUIR PRIN 2006 research project is gratefully acknowledged. 
[1] E.A: Cornell and C.E. Wieman, Rev. Mod. Phys., 74, 875 (2002).

[2] W.Ketterle, Rev. Mod. Phys. 74, 1131 (2002).

[3] B. DeMarco and D.S. Jin, Science, 285, 1703 (1999).

[4] A.G. Truscott, K.E. Stricker, W.I. McAlexander, G.B. Partridge and R.G. Hulet, Science, 291, $2570(2001)$.

[5] M. Baranov, L. Dobrek, K. Garāl, L. Santos and M. Lewenstein, Phys. Scr. T102, 74 (2002).

[6] D. De Mille, Phys. Rev. Lett., 88, 067901 (2002).

[7] e.g. see: H.L. Bethlem and G. Meijer, Int. Rev. Phys. Chem., 22, 73(2003).

[8] N. Balakrishnan, R.C. Forrey and A. Dalgarno, Chem. Phys. Lett., 279, 50, (1997).

[9] N. Balakrishnan, R. Forrey and A. Dalgarno, Chem. Phys. Lett., 280, 1 (1997).

[10] e.g. see: E. Bodo, F.A. Gianturco , Int. Rev. Phys. Chem., 25, 313 (2006).

[11] R.V. Krems, Int. Rev. Phys. Chem., 24, 99 (2005).

[12] N. Zahram, T. Vogt, M. Mudrich, D. Comparat and P. Pillet, Phys. Rev. Lett., 96, 023202 (2006).

[13] E. Bodo, F.A. Gianturco, Phys. Rev. A, 73, 052715 (2006).

[14] E. Bodo, F.A. Gianturco, Europhys. Lett., 77, 33001 (2007).

[15] E. Bodo, F.A. Gianturco and R. Martinazzo, Chem. Phys., 271, 309 (2001).

[16] R. Martinazzo, E. Bodo, F.A. Gianturco and M. Raimondi, Chem. Phys., 287, 335 (2003).

[17] T. Stoecklin and A. Voronin, Phys. Rev. A, 72, 042714 (2005).

[18] P.E. Wigner, Phys. Rev. 73, 1002 (1948).

[19] for a recent analysis see: F.A. Gianturco and K. Willner, Phys. Rev. A 75, 062714 (2007).

[20] W. Kolos and L. Wolniewicz, J. Chem. Phys., 43, 2429 (1965)

\section{List of Figures}

1 Collinear minimum energy path profile of the $\mathrm{Li}^{+}+\mathrm{H}_{2}$. The molecular energy levels accessible below the reactive barrier are also reported (all energies in $\mathrm{cm}^{-1}$ and distances in $\AA$ ). Adapted from Ref. [14 . . . . . . . . . . . . . . 14 
2 Computed coupling matrix elements (diagonal terms) between $\mathrm{H}_{2}$ vibrational levels and for two different approaches of the lithium ion. Left panel: collinear approach; right panel: T-shaped approach. . . . . . . . . . . . . . 15

3 Computed elastic cross sections for the $\mathrm{Li}^{+}+\mathrm{H}_{2}(v)$ system as a function of collision energy and for a sequence of vibrationally excited initial state of the molecule. See text for computational details. . . . . . . . . . . . . . . . . 16

4 Computed quenching cross sections for collisional de-excitations from different initial states and for the $(\Delta v=-1, \Delta j=+2)$ process. The dominant contributions of $\mathrm{J}=0$ (dashes) and $\mathrm{J}=1$ (dots) are shown for each integral cross section (solid lines). . . . . . . . . . . . . . . . . . . . . 17

5 Computed total quenching cross sections (vibrational) from different initial $(v, 0)$ states of the $\mathrm{H}_{2}$ molecule. See text for details. . . . . . . . . . . 18

6 Computed scattering attributes as a function of the initial vibrational level preparation of the $\mathrm{H}_{2}$ partner. Upper left panel: total inelastic cross section maxima around the $10^{-3} \mathrm{~cm}^{-1}$ region. Upper right panel: sign and value of the real part of the scattering length as a function of $v$. Lower left panel: quenching rate coefficients from eq.(10). Lower right panel: locations of bound and virtual states as s function of initial $v$ state. . . . . . . . . 19

\section{Figures}




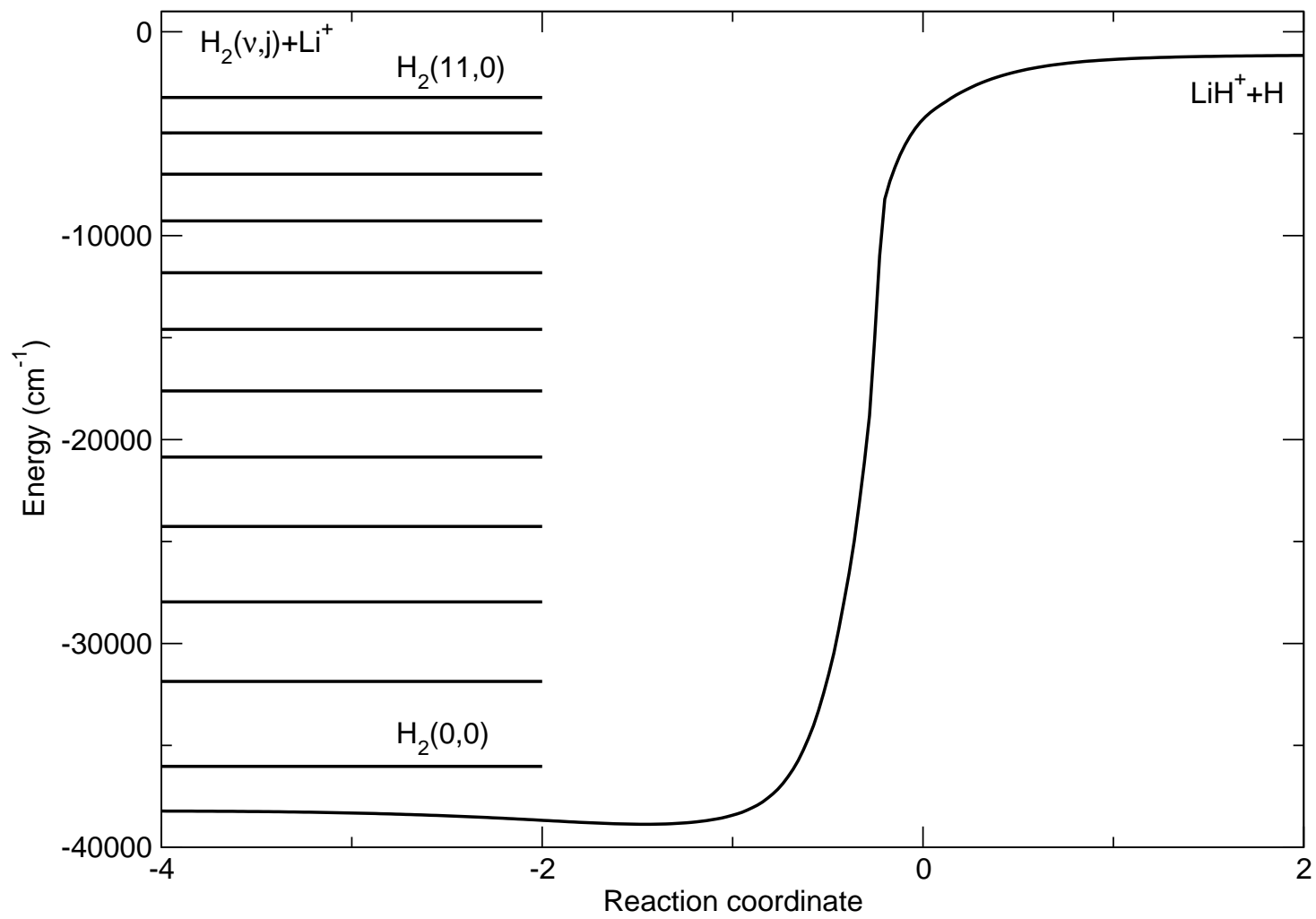

FIG. 1: Collinear minimum energy path profile of the $\mathrm{Li}^{+}+\mathrm{H}_{2}$. The molecular energy levels accessible below the reactive barrier are also reported (all energies in $\mathrm{cm}^{-1}$ and distances in $\AA$ ). Adapted from Ref. [14]. 

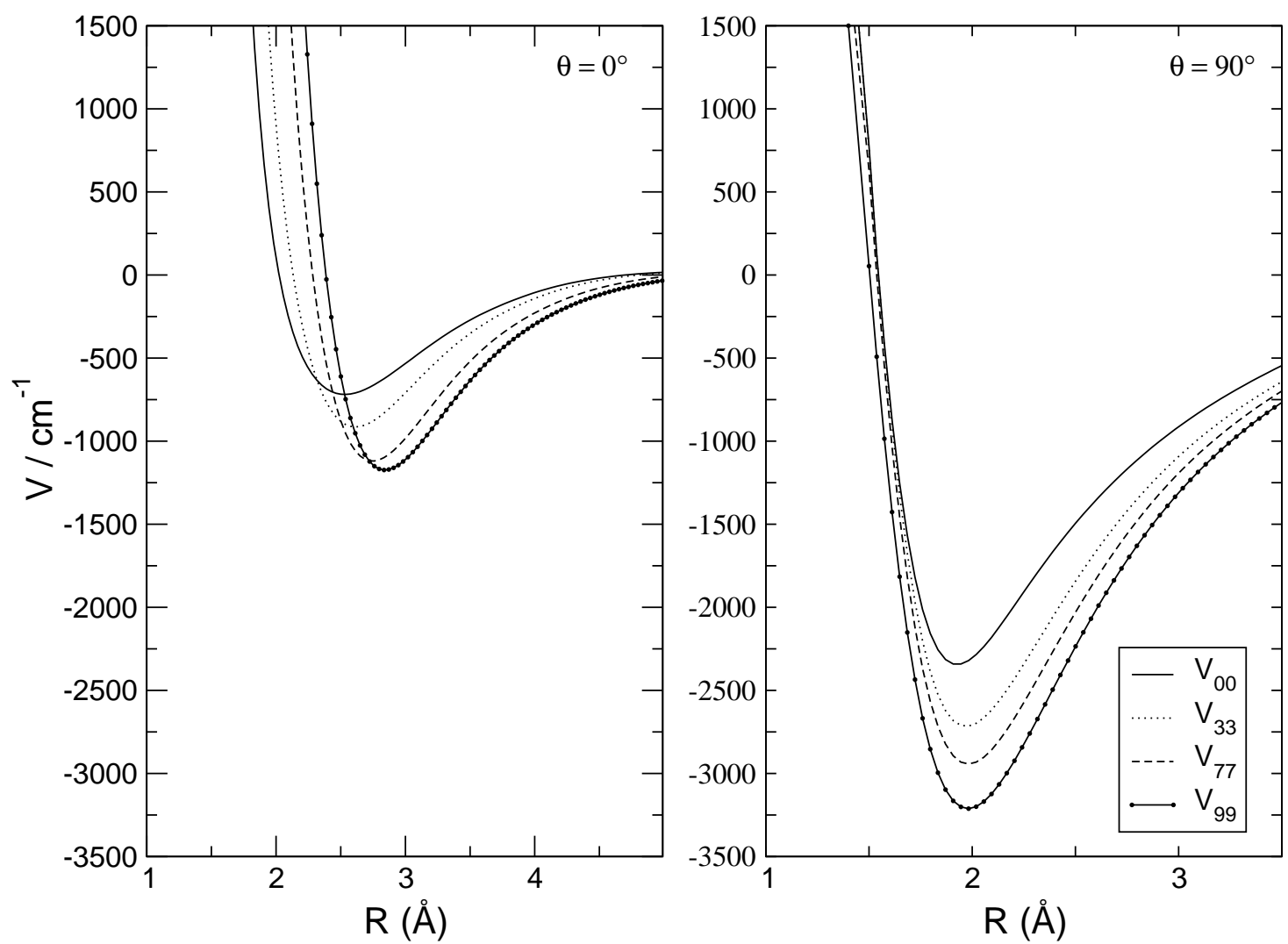

FIG. 2: Computed coupling matrix elements (diagonal terms) between $\mathrm{H}_{2}$ vibrational levels and for two different approaches of the lithium ion. Left panel: collinear approach; right panel: T-shaped approach. 

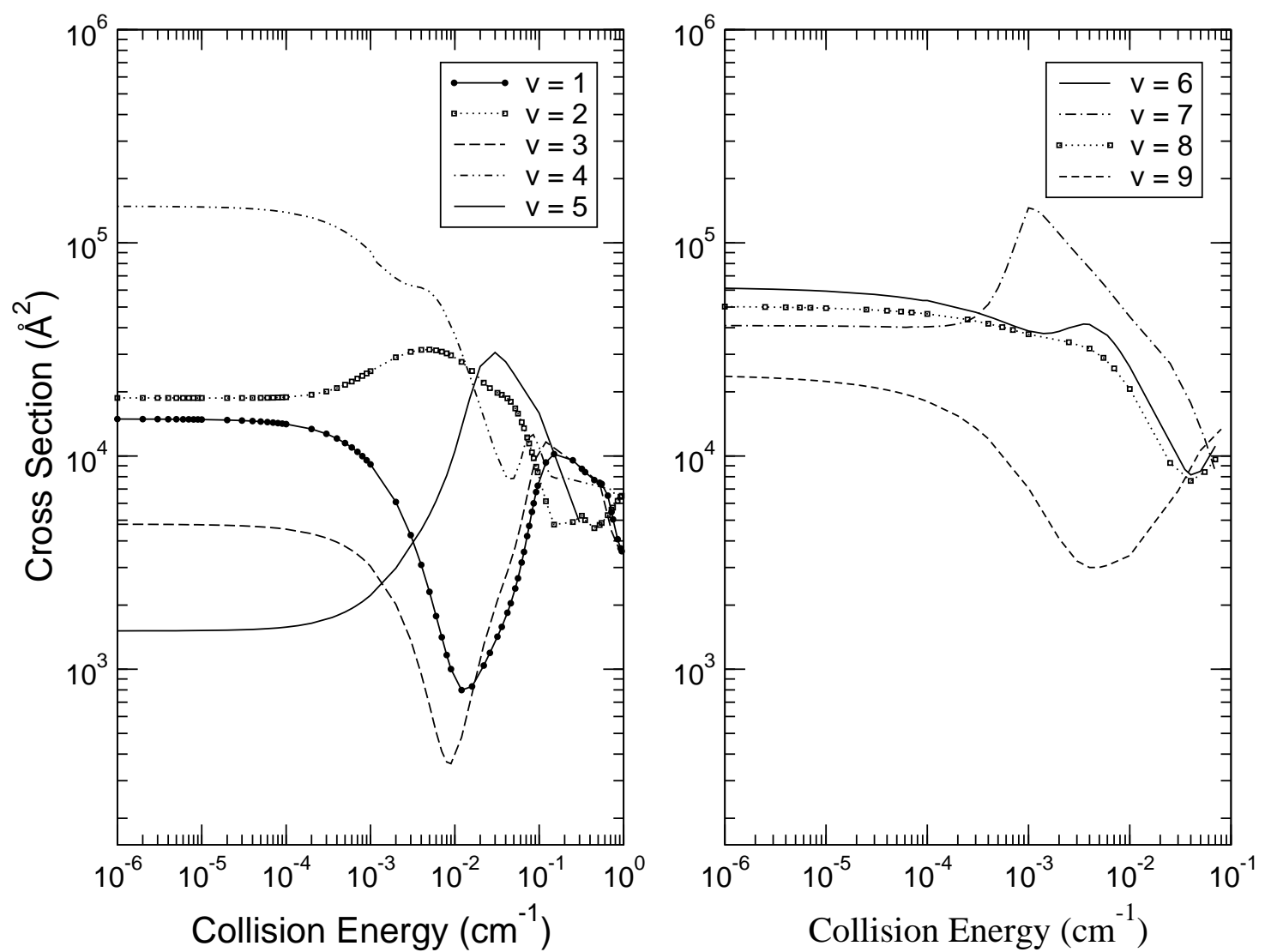

FIG. 3: Computed elastic cross sections for the $\mathrm{Li}^{+}+\mathrm{H}_{2}(v)$ system as a function of collision energy and for a sequence of vibrationally excited initial state of the molecule. See text for computational details. 

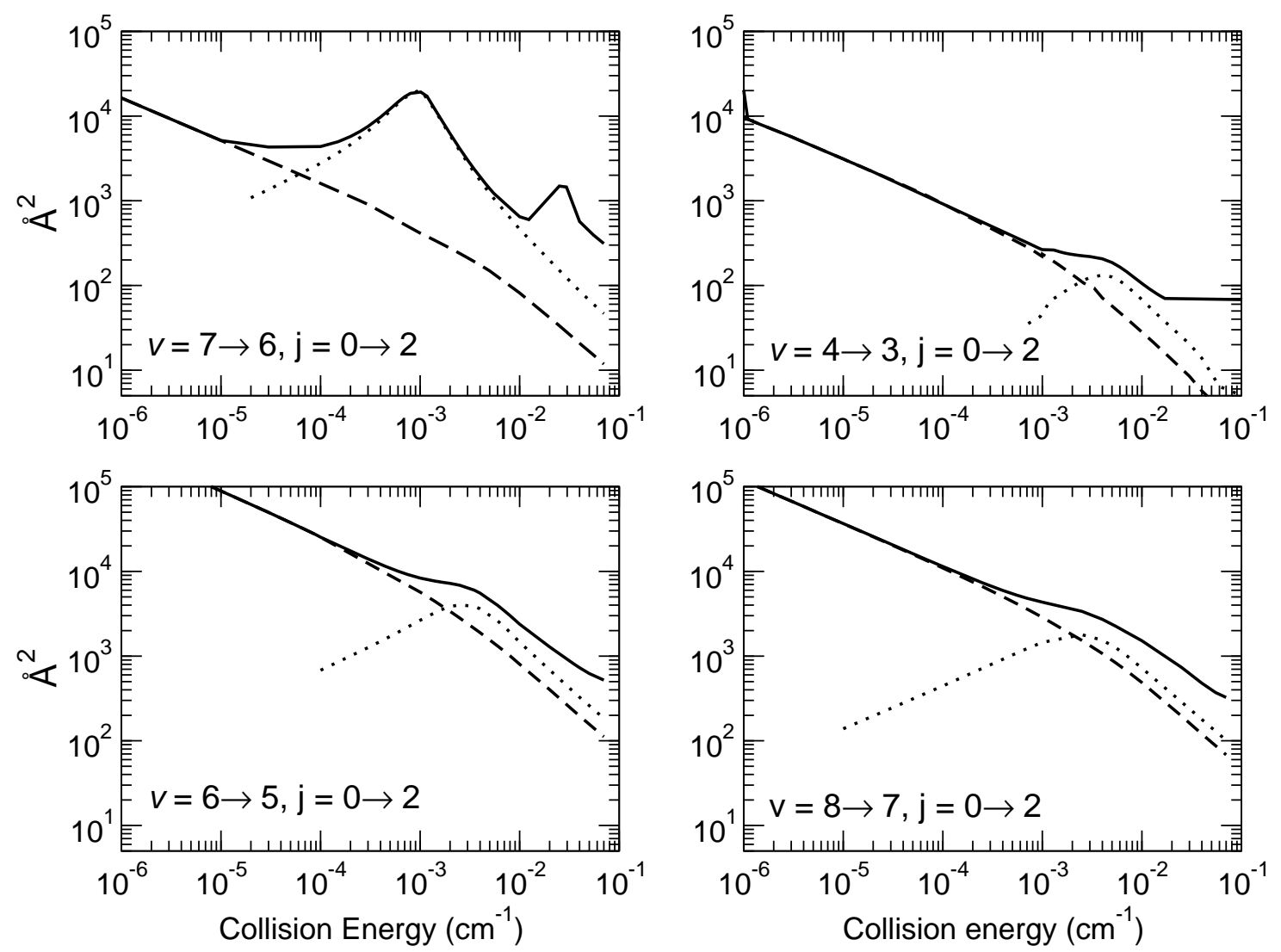

FIG. 4: Computed quenching cross sections for collisional de-excitations from different initial states and for the $(\Delta v=-1, \Delta j=+2)$ process. The dominant contributions of $\mathrm{J}=0$ (dashes) and $\mathrm{J}=1$ (dots) are shown for each integral cross section (solid lines). 

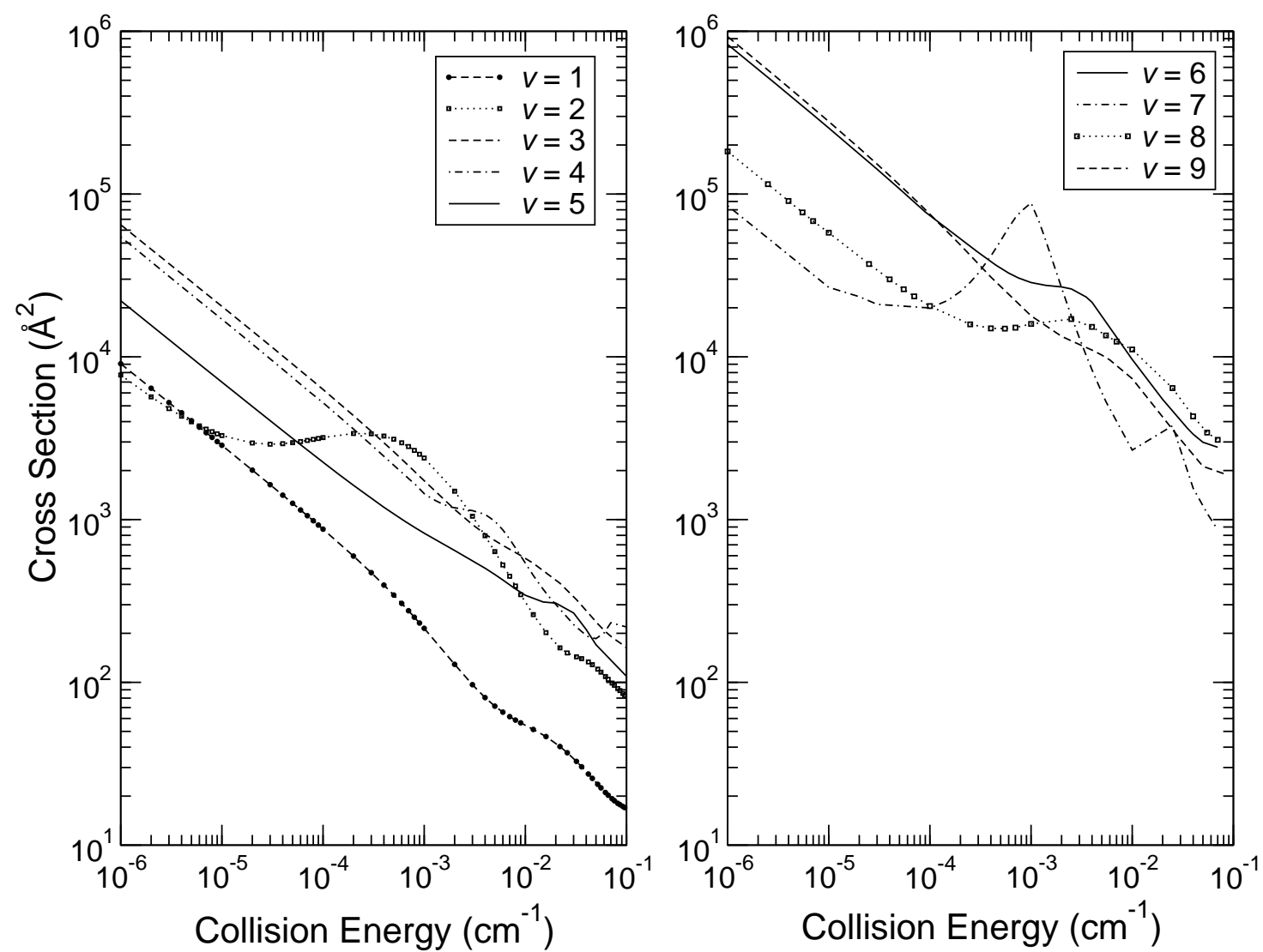

FIG. 5: Computed total quenching cross sections (vibrational) from different initial $(v, 0)$ states of the $\mathrm{H}_{2}$ molecule. See text for details. 

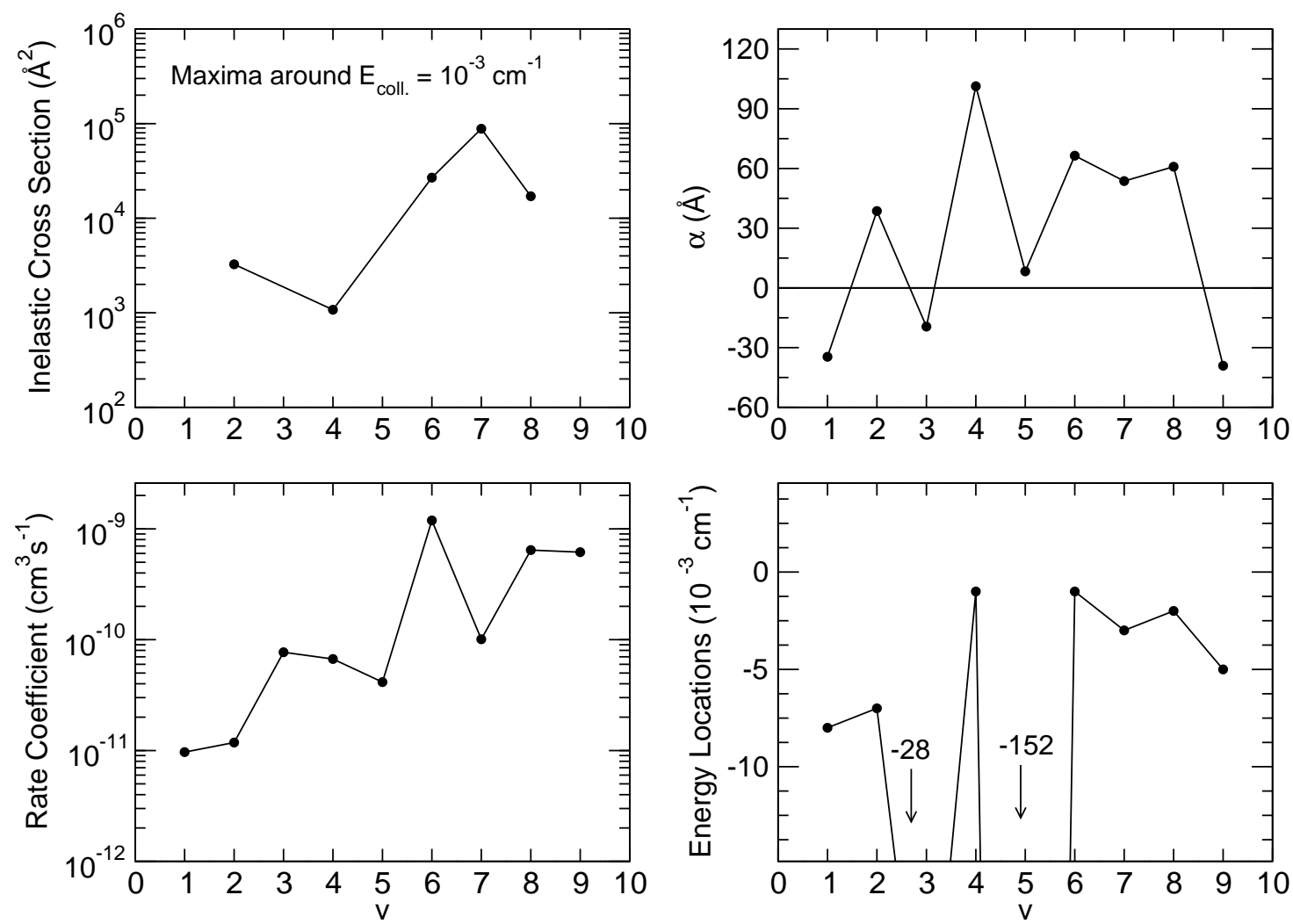

FIG. 6: Computed scattering attributes as a function of the initial vibrational level preparation of the $\mathrm{H}_{2}$ partner. Upper left panel: total inelastic cross section maxima around the $10^{-3} \mathrm{~cm}^{-1}$ region. Upper right panel: sign and value of the real part of the scattering length as a function of $v$. Lower left panel: quenching rate coefficients from eq.(10). Lower right panel: locations of bound and virtual states as s function of initial $v$ state.

Tables

\begin{tabular}{ccccccc}
\hline \hline$v$ & $\alpha(\AA)$ & $\beta(\AA)$ & $\mathrm{E}\left(10^{-3} \mathrm{~cm}^{-1}\right)$ & $\Gamma(\mathrm{ns})$ & $R_{v}(T \rightarrow 0)\left(\mathrm{cm}^{3} \cdot \mathrm{s}^{-1}\right)$ & $R_{v}\left(\mathrm{~cm}^{3} \cdot \mathrm{s}^{-1}\right)$ \\
\hline \hline 0 & 36.90 & 0.0 & -8 & $\infty$ & 0.0 & - \\
1 & -34.60 & 0.22 & -8 & 23 & $1.12 \cdot 10^{-11}$ & - \\
2 & 38.60 & 0.18 & -7 & 39 & $9.17 \cdot 10^{-12}$ & - \\
3 & -19.46 & 1.65 & -28 & 0.5 & $8.42 \cdot 10^{-11}$ & - \\
4 & 101.3 & 1.31 & -1 & 97 & $6.69 \cdot 10^{-11}$ & -
\end{tabular}




\begin{tabular}{ccccccc}
5 & 8.30 & 0.81 & -152 & 0.09 & $4.14 \cdot 10^{-11}$ & - \\
6 & 66.43 & 23.3 & -1 & 2 & $1.19 \cdot 10^{-9}$ & $1.50 \cdot 10^{-9}\left(10^{-3} K\right)$ \\
7 & 53.65 & 1.99 & -3 & 9.5 & $1.01 \cdot 10^{-10}$ & $1.70 \cdot 10^{-9}\left(10^{-3} K\right)$ \\
8 & 60.87 & 12.6 & -2 & 2.4 & $6.43 \cdot 10^{-10}$ & $1.22 \cdot 10^{-9}\left(10^{-2} K\right)$ \\
9 & -39.07 & 12.0 & -5 & 0.7 & $6.16 \cdot 10^{-10}$ & - \\
\hline \hline
\end{tabular}

TABLE I: Real $(\alpha)$ and imaginary $(\beta)$ components of the scattering lengths, energy locations (E) and lifetimes $(\Gamma)$ of the virtual and bound states, and quenching rates $\left(R_{v}\right)$ from the considered $v$ initial levels of $\mathrm{H}_{2}$. The rates c(the rotational initial state is $j=0$ )omputed in the millikelvin regimes are shown in the last column for a few of the initial target states. 